V. Aus der III. medicinischen Klinik und Universitätspoliklinik des Herrn Geh. Med.-Rath Prof. Dr. Senator.

\title{
Ueber die chirurgische Behandlung der Magenkrankheiten. ${ }^{1}$ )
}

Von Dr. 'Th. Rosenhein, Privatdocenten und I. Assistenten.

Der Aufschwung, den die Magenchirurgie genommen hat, gebört der jüngsten Zeit an, er datirt recht eigentlich von dem im Jahre 1881 gelungenen Versuche Bill roth's, einen carcinomatösen Pylorus zu entfernen. Betrachtete nıan bis dahin die Behandlung. der Magenkrankheiten als die Domäne der inneren Medicin, so änderte sich nach diesem erwähnten und anderen Erfolgen die Meinung; nicht blos bei bösartigen Neubildungen wollte der Operateur eingreifen, sondern auch die gutartigen Stenosen, die idiopathische Erweiterung des Organs, das Ulcus mit seinen Complicationen sollten seinen therapeutischen Bestrebungen zugänglich gemacht sein, und vielleicht betrachten annexionslustige Clirurgen diese Kranklueiten bereits als solche, die in erster Roihe vor ihr Forum gehören.

Erfolge wurden zweifellos auf dem operativen Wege errungen; es ist in der Vervollkommnung der Technik in kurzer Zeit Glänzendes geleistet worden, und für einzelne Krankheitsfälle kann man gute Resultate als mit Sicherheit erreichbar hinstellen. Nicht zum wenigsten sind diese werthvollen Ergebnisse erzielt worden durch die feinere Diagnostik der Magenkrankheiten, die die Untersuchungen der letzten Jahre kennen gelehrt haben. Auf keinem Gebiete hat sich der Nutzen der Zusammenarbeit der inneren Mediciner und Chirurgen mehr gezeigt, als auf dem der Magenkrankheiten, und auf keinem sind die Vertreter beider Richtungen mehr aufeinander angewiesen, als auf diesem.

Besteht nun diese gegenseitige nahe Beziehung, so ist es klar, dass für den Arzt, der ein in Rede stehendes Magenleiden vom internen Standpunkte aus beurtheilt, die Frage von der grössten Wichtigkeit ist: Wann bedarf ich des Chirurgen? - mit anderen Worten, uns interessirt im höchsten Maasse die Indicationstellung für den chirurgischen Eingriff! Nun ist dieser wichtigen Angelegenheit in einigen Lehrbüchern (Debove et Raymond, Boas u. a.) zwar bereits Berücksichtigung zutheil geworden, und Guinard ${ }^{2}$, $\mathrm{Mintz}^{3}$ ) und anderen verdanken wir treffliche Abhandlungen, in denen auf Grund einer weither zusammengetragenen Casuistik die uns beschäftigende Frage für verschiedene Magenkrankheiten discutirt wird, aber gemeinhin hat man bei diesen Autoren den Eindruck, dass sie zu ihrem Urtheil gelangen, indem sie das. Facit aus den Erfahrungen anderer ziehen, da ihre eigene zur Entscheidung unzureichend ist.

Es ist auch merkwürdig, dass, während die Chirurgen auf zahlreichen Congressen sich über ihre therapeutischen Ergebnisse Rechenschaft ablegten, während die inneren Kliniker bei gleichen Gelegenheiten, über die Bestrebungen, das diagnostische Rüstwerk zu verbessern, berichteten, diese Frage, die von so ausserordentlicher Bedeutung ist, noch niemals zur öffentlichen Discussion kam.

Da ich nun glaube, dass das Material, das mir die letzten Jahre an die Hand gegeben haben, wohl gross genug ist, um selbstständig ein Urtheil $\mathrm{zu}$ gewinnen und in strittigen Punkten Stellung nehmen zu können, so ergreife ich hier das Wort in dieser Sache. Ohne gerade eine endgültige Entscheidung herbeizuführen, werden wir doch, wie ich denke, nach dent heutigen Stande der Dinge zu einem brauchbaren Ergebniss bei der Discussion dieser von mir aufgerollten Frage kommen können, wenn wir unsere Erfahrungen zusammenfassen. Ich bin ferner der Ansicht, dass es Zeit ist, dass wir der Angelegenheit näher treten, da es mir scheint, dass Uebergriffe von chirurgischer Seite stattfinden, indem Krankheitszustände operativ behandelt werden, die auf anderem Wege ohne jede Gefahr zu bessern und zu heilen sind. Und ich darf es schliesslich wohl sagen, dass unsere Gesellschaft ganz besonders berufen ist, hier ihr Wort zu erheben, zumal der uns heute beschäftigende Gegenstand bei zahlreichen Demonstrationen an dieser Stelle bereits gestreift worden ist.

Es war von vornherein gegeben, dass man die Hülfe des Chirurgen am ehesten bei der Bekämpfung der bösartigen Geschwulstbildung suchte, und hier wurde auch durch Billroth der erste grosse Erfolg errungen. Kein Arzt ist heute im Zweifel, dass, wenn er ein Carcinom an irgend einem Organ, an der Haut, an den Schleimhäuten, an den Drüsen findet, er es in erster Reihe von dem Standpunkte aus zu betrachten hat: welche Chance bietet es für die Radicaloperation? In betreff der Magenkrebse aber steht diese wichtige Erwägung bei vielen noch nicht im Vordergrunde, indem Unkenntniss dessen, was geleistet werden

1) Vortrag, gehalten im Verein für innere Medicin am 29. Oct. 1894.

2) Traitement chirurgical du cancer de l'estomac. Paris 1892.

$3)$ Zeitschr. f. kl. Medicin Bd. XXV, S. 123. 
kann, oder auch übertriebener Skepticismus diesen Gedauken nicht aufkommen lassen. Und doch haben wir einen Erfolg diesem mörderischen Uebel gegenüber nur voll der Hand des Clilıu'gen zu erwarten, und es wird thatsächlich schon heute von berufener Seite rühmend anerkaunt, dass die Egebnisse in der chirurgischen Behaudlung des Magenkrebses kaum weniger günstig sind als die, die von den glücklichsten Operateuren bei den Carcinomell anderer Körpertheile erzielt wordel sind (Kraskel)). Es ist deshalb der Gesichtspunkt der chirul'gischen Hülfe hier mit demselben Rechte, wie z. B. beim Mastdarmcarcinom, wo er wohl keiner Discussion niehr unterliegt, an die erste Stelle zu rücken: jeder Fall von Magenkrebs ist vor allem daraufhin anzusehen, ob er sich für die Radicalbehandlung eignet. Daran äludert die Thatsache, dass eine dauernde Heilung in keinem bisher operirten Falle verbürgt ist, gar nichts. Denn die Magenresection hat erst zu kurze Zeit Bürgerrecht erworben, als dass wir über den Ausgang aller unter günstigen Bedingungen und mit allen Cautelen operirten Fälle schon heute etwas sagen könnten. Immerhin kennen wir verschiedene Fälle, die fiinf Jahre recidivfrei blieben; meine Patientin Paul, die ich ${ }^{2}$ Ihnen jüngst wieder vorstellte, befindet sich fast vier Jahre nach der Resectio pylori noch immer vorzüglicl, und ich zweifle nicht, dass wir beim Magencarcinom zu derselbeı Grenze der Leistungsfähigkeit gelangen werden, wie bei irgend welchen anderen Sclleimhautkrebsen, bei denen bekanntlich endgültige Heilerfolge auch selten genug sind.

Aber niemand wird an der Berechtigung des chirurgischen Eingriffs zweifeln, niemand den Werth desselben herabsetzen, auch wenn wir nichts besseres zu erzielen vermöchten, als die Kranken für eine beschränkte Anzahl Jahre gesund und leistungsfähig zu machen. Ja, ich glaube, Arzt und Patient werden eine Operation noch als segensreich anerkennen müssen, wenn sie ïberhaupt nur dazu angethan ist, in dem trostlosen Zustande, in dem sich der Krebskranke befindet, zu nützen. Selbst wo wir nicht mehr radical vorgehen können, wird jede Palliativoperation berechtigt sein, wenn wir den unsäglichen Qualen derartiger Kranker ein Ende bereiten können, ohne sie bei der Operation einer Gefahr auszusetzen, die im Verhältniss zum gestifteten Nutzen zu gross erscheint.

Damit kommen wir zu der wichtigen Erörterung der Gefahr, welche die Kranken beim operativen Vorgehen laufen. Die chirurgische Behandlung des Magenkrebses erstreckt sich, genall genommen, auf die Carcinome am Pylorus und in unmittelbarer Nähe desselben; denn sitzt die Neubildung an anderer Stelle, so kommt nur selten eine Operation in Betracht. Einmal ist sie dann gar nicht mehr oder nur bei stark dislocirtem Magen für das Messer angreifbar, andererseits macht sie auch nur selten, z. B. an der Cardia Störungen, zu deren Abhülfe wir genöthigt wären an den Chirurgen zu appelliren. Es scheiden mithin von vornherein etwa $40^{\circ}, 0$ aller Magenkrebsfälle aus, für die wir auf die Heilfactoren, die uns die innere Medicin an die Hand giebt, angewiesen bleiben. Für die iibrig bleibenden $60^{\circ}$ kommen im wesentlichen zwei Operationen in Frage, die wir auf ihre Gefahren zu prüfen liaben: die Resection, die eine radicale Beseitigung des Uebels herbeiführen soll, und die Gastroenterostomie, die als Palliativverfahren von grösstem Werthe ist, indem sie bei vorhandener Stagnation für einen rechtzeitigen und leichten Uebertritt der Ingesta ins Jejunum mit Umgehung des Duodenum sorgt. Betrachten wir diese beiden Operationen gesondert - und da sie nicht gleichwerthig in Erfolge und verschieden in Bezug auf ihre Schwere sind, müssen wir dies wohl - so finden wir, wenn wir die Statistik befragen, folgendes:

Bei der Resection starben nach der Zusammenstellung von ( u inard, ${ }^{3}$ ) der alle Fälle bis zum Jahre 1891 berücksichtigt, von 153 Kranken $96=62 \%$; es zeigt sich ferner hierbei, dass in dem Zeitraum bis zum Jahre 1887 die Mortalität $71 \%$, in dem folgenden bis zum Jahre 1891 nur noch $52 \%$ betrug. Aber eine solche zusammenfassende Statistik, bei der die Sünden und Ungeschicklichkeiten des einen einem anderen erfahrenen und vorsichtigen Operateur mit angerechnet werden, giebt uns kein richtiges Bild; klarer sehen wir schon, wenn wir die Ergebnisse einzelner hervorragender Chirurgen verwerthen. Billroth ${ }^{4}$ ) verlor von 29 Kranken $16=55 \%, \mathrm{Kocher}^{5}$ ) starben von 9 Kranken $2=22 \%$, aber er bemerkt ausdrücklich, dass die sechs zuletzt operirten glatt durchkamen; die gleiche Erfahrung haben Kraske6) und $\mathrm{Hahn}^{7}$ ) mitgetheilt: auch bei ihnen sind die Resultate von Jahr zu Jahr günstiger geworden.

1) Berl. kl. Wochenschr. 1894, No. 20, 21.

2) Deutsche med. Wochenschr. 1894, No. 30.

3) Traitement chirurgical du cancer de l'estomac. Paris 1892.

4) Verhandlungen des X. internationalen Congresses, Bd. III.

5) Correspondenzblatt für Schweizer Aerzte 1893

6) Berliner klin. Wochenschr. 1894, No. 20, 21.

7) Deutsche med. Wochenschr. I894, No. 43.
Bei der Gastroenterostomie starben nach der zusammeufassenden Statistik von Guinard bis zum Jalre 1891 von 105 Krebskranken $33=31 \%$. Rockwitz ${ }^{1}$ ) giebt auf der Strassburger Klinik nur eine Mortalitätsziffer von $12^{1 / 2} \%$ an. Hahn hat in der letzten Zeit 15 mal lintereinander die Gastroenterostomie gemacht, ohne einen Kranken zu verlieren; in etwa $3 / 4$ seiner Fälle handelte es sich um Krebs.

Aus diesen Angaben lässt sich wohl das entnehmen: nichts ist trügerischer, als solch eine zusammenfassende Statistik, wo es sich um die Entscheidung über den Werth eines Verfahrens handelt, das noch in der Ausbildung begriffen ist, das an das specialtechnische Können so überaus hohe Anforderungen stellt. Berücksichtigen wir: es giebt wohl wenige Operateure, die mehr als zehn Magenresectionen ausgeführt haben, und auch die hervorragendsten unter ihnen bedürfen einer besonderen Erfahrung und Einarbeitung auf diesem Gebiete, bevor sie mit geniigender Sicherheit die Schwierigkeiten, die ihnen bei ihrem Vorgehen begegnen und die die Nachbehandlung bietet, beherrschen lernen. So lange diese Fähigkeit nicht erworben ist, laufen die Kranken unzweifelhaft grössere Gefahr; ist sie aber vorhanden und vereint sie sich mit einer richtigen Indicationsstellung in der Auswahl des operativen Verfahrens, so sind die Gefahren gering. Wir müssen auch bedenken, dass eine grosse Zahl von Operationen an ganz ungeeigneten Fällen gemacht wird: Die Kranken und gewisse Umstände drängen zu einem operativen Versuch, wo der vorsichtige Arzt keinen Erfolg ill Aussicht stellen kann. Wie diese Momente zusammeuwirken, um eine Statistik zu beeinflussen und geradezu wertllos zu machen, werden Thnen die Zahlen, die ich aus eigener Erfahrung gewonnen habe, zeigen.

Scheide ich einen Fall aus der Privatpraxis (Frau K., 64 Jahre alt, operirt am 26. Mai 1892) aus, bei dem die Loreta'sche Operation gemacht wurde, aber ungünstig ablief, so habe ich noch über 15 Magenkrebskranke zu berichten, bei sieben wurde die $R e-$ section, bei acht die Gastroenterostomie gemacht. Von den sieben ersterwähnten sind an der Operation resp. ihren unmittelbaren Folgen drei gestorben, und zwar ein Mann von-46 Jahren, über den ich bereits früher ${ }^{2}$ ) berichtete, an einer Blutung aus einem Stichcanal nach 24 Stunden, eine 63jährige Frau nach 48 Stunden im Collaps, eine 37jährige Frau am neunten Tage an Pneumonie. Der zuerst erwähnte Ungliicksfall passirte vor zwei Jahren, er ist der operativen Technik zur Last zu legen. Im zweiten Fall handelte es sich um ein aufs äusserste heruntergekommenes Individuum mit faustgrossem, aber gut beweglichem Pyloruscarcinom. Der Eingriff wurde mit geringer Hoffnung auf Drängen der Kranken unternommen; vielleicht wäre hier doch nocl ein theilweiser Erfolg erzielt worden, hätte man sich mit der Gastroenterostomie begnügt, aber die Leichtigkeit, mit der der grosse Tumor abgrenzbar war, das Fehlen von Metastasen war für den Operateur zu verführerisch. Im dritten Falle bestand von vornherein eine leichte Bronchitis, die sich ganz allmählich, trotzdem die Wundheilung vorzïglich von statten ging und trotzdem die Kräfte der Kranken bereits zugenommen hatten, zu einer Pueumonie entwickelte; diese Zufälligkeit können wir bei keiner grösseren Operation auschliessen, der Ausgang fällt nicht dem speciellen chirurgischen Eingriff zur Last. Von den vier Kranken, die die Operation überstanden, kennen Sie Fräulein Paul, die im Februar 1891 operirt wurde, und Fraı Weissenborn, bei der der Krebs am 4. März d. J. entfernt wurde; beiden geht es vortrefflich. Frau Weissenborn hat in diesem Jahre 41 Pfund zugenommen. Der dritte Kranke, der die Operation überstand, ist etwas länger als ein Jahr am Leben geblieben, er starb, nachdem er sich geraume Zeit vortrefflich befunden hatte, an maligner Peritonitis. Auch die jüngst Operirte hat sich bereits ausserordentlich erholt.

Name $\quad$ Alter $\left|\begin{array}{c}\text { Datum der } \\ \text { Operation }\end{array}\right|$ Ergebniss

\begin{tabular}{llll|l|l} 
1. Paul . . . & 46 Jahre & 11. Febr. 1891 & Votzligliches Befinden. \\
2. Lübeck &. & . & 46 Jahre & 30. Juni 1892 & Gestorben 1. Juli 1892 an Blu-
\end{tabular}

3. Klett . . 37 Jahre 13. Febr. 1893 Gestorben 22. Februar 1893 an

40 Jahre 3. Mai 1893 Bronchopneumonie.

1893 Gestorben 13 Monate später

5. Weissenborn 45 Jahre 4 Mälz 1894 Zur Zeit gutes Befinden (41 Pfd. 6. Link 63 Jahre 14. April 1894 Körpergewichtszunahme). Collaps.

7. Stock. . . 48 Jahre 5. Nov. 1894 Rasche Erholung (bis 12. Dezember 1894 beobachtet; $\mathrm{Zu}$ satz b. d. Correctur).

1) Zeitschr. f. Chirurgie, Bd. XXV, S. 524.

2) Deutche med. Wochenschr. 1894, No. 30 . 
Von den acht Krebskranken, bei denen die Gastroenterostomie ausgefïhrt wurde, ist keiner zugrunde gegangen. Alle erholten sich rasch, wurden mehr oder weniger ganz beschwerdefrei und nahmen an Körpergewicht zum Theil erheblich (bis zu 30 Pfund) zu. No. 2 befand sich länger als acht Monate ganz gut. In zwei Fällen, No. 4, 6, trat der Tod schon nach drei Monaten ein. Bei No. 1 habe ich über den Ausgang nichts erfahren können. Vier Patienten leben noch bei zum Theil vortrefflichem Allgemeinzustand, sie befinden sich fünf bis neun Monate nach der Operation. No. 3

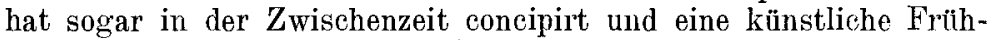
geburt'1) gut überstanden. Will man diese Ergebnisse recht würdigen, so muss man bedenken, dass es sich ausnahmslos um vorgeschrittene bösartige Processe handelte, bei denen der Eingriff unternommen wurde: alle Kranken waren im Stadium schwerster Unterernährung, sie waren durch hartnäckiges Erbrechen und anhaltende Schmerzen bedeutend heruntergekommen.

\begin{tabular}{|c|c|c|c|}
\hline $\mathrm{Name}$ & & Alter & Datum der Operation \\
\hline $\begin{array}{l}\text { 1. Lintow } \\
\text { 2. E. : : } \\
\text { 3. Baumgart : } \\
\text { 4. L. } \\
\text { 5. Polensky : : } \\
\text { 6. Siebert : } \\
\text { 7. Musculus : } \\
\text { 8. Glawe. }\end{array}$ & $\begin{array}{ll} & : \\
\therefore & : \\
\therefore & : \\
& :\end{array}$ & $\begin{array}{l}56 \text { Jahre } \\
40 \text { Jahre } \\
\text { 31 Jabre } \\
40 \text { Jahre } \\
49 \text { Jahre } \\
50 \text { Jahre } \\
55 \text { Jahre } \\
51 \text { Jahre }\end{array}$ & $\begin{array}{l}\text { 19. September } 1891 \\
\text { 21. Februar } 1893 \\
\text { 6. Februar } 1894 \\
\text { 11. Februar } 1894 \\
\text { 24. Mai } 1894 \\
\text { 4. Juni } 1894 \\
\text { 12. Juni } 1894 \\
\text { 21. Juni } 1894\end{array}$ \\
\hline
\end{tabular}

Betrachten wir also die Statistik mit Kritik und berücksichtigen wir auch die Ergebnisse gesonderter Zeitabschnitte, so haben wir Erfreuliches von den gemachten Fortschritten zul berichten. Es erhellt schon aus den Mortalitätsziffern, dass die Resection die gefährlichere Operation ist: sie dauert länger (zwei bis drei Stunden und mehr), sie stellt demgemäss höhere Anforderungen an die Widerstandsfähigkeit des Kranken, und ihre Technik ist schwieriger. Gefahr la ufen die Kranken in mer, und nur wenn man die allergünstigsten Fälle für diesen Eingriff reservirt, ist das Risico nicht grösser als bei anderen eingreifenderen Operationen wegen Krebs. Dafür hat der Kranke die Chance, nicht luur von seinem augenblicklichen Leiden befreit, sondern auch für geraume Zeit gesund und arbeitsfähig, vielleicht sogar dauernd geheilt zu werden. Wir werden das chirurgische Vorgehen befürworten, wenn der Fall aussichtsvoll ist, d. h. wenn der Patient nicht zu sehrheruntergekommen ist und der lokale Befund günstige Erwartungen erweckt. Einigermaassen leicht ist es, ein Urtheil über die verfügbaren Kräfte des Patienten zu gewinnnen: hier wird der Znstand des Allgemeinbefindens, der Grad der Abmagerung und vor allem der Puls einen Maassstab an die Hand geben. Enorm schwer ist es dagegen, ein richtiges Bild von dem Umfang der carcinomatösen Erkrankung vor Eröffnung der Bauchhöhle zu erhalten. Man täuscht sich leicht über die Grösse der krebsigen Wucherung; Adhäsionen und Metastasenbildung können sich dem Nachweis auch bei gewissenhaftester Untersuchung ganz entziehen. Unter den scheinbar günstigsten Fällen erweisen sich nachträglich noch recht viele als ungeeignet für die Resection. Als diese Operation contraindicirend sind anzusehen: ausserordentliche Grösse der Neubildung, so dass mehr als die Hälfte des Magens entfernt werden müsste, ferner ausgedehnte Verwachsungen mit Leber oder Pancreas, Infection benachbarter Organe, speciell auch des Netzes, endlich meist auch Anschwellung der Drüsen, obgleich die Tumescenz derselben selbst in der Nähe des carcinomatösen Heerdes noch nicht mit Sicherheit für ihre maligne Erkrankung spricht.

(Fortsetzung folgt.) 